\title{
Report of isolated epidural extra-nodal Rosai-Dorfman disease of cervicothoracic spine
}

\author{
Apoorv Kumar ${ }^{1}$ Balamurugan Thirugnanam ${ }^{1} \cdot$ Shaffaf Abdul Kareem $\mathbb{D}^{1} \cdot$ S. P. Ajay Kumar ${ }^{1} \cdot$ S. Vidyadhara ${ }^{1}$
}

Received: 29 June 2020 / Revised: 11 August 2020 / Accepted: 12 August 2020

(C) International Spinal Cord Society 2020

\begin{abstract}
Introduction Rosai-Dorfman disease (RDD) or sinus histiocytosis with massive lymphadenopathy is a nonneoplastic lymphoproliferative disorder which commonly presents with painless massive cervical lymphadenopathy along with fever and weight loss. Isolated extra-nodal disease involvement, especially isolated central nervous system disease without lymph node involvement is rare.

Case presentation A 35-year-old man presented with upper back pain and paraparesis of 2 months duration. Contrast-MRI revealed an enhancing epidural mass lesion from C6-T7 suggestive of a primary epidural spinal-cord tumor. The lesion was resected and posterolateral fusion C6-T8 was performed. Histopathological examination revealed an isolated extra-nodal case of RDD. He was started on chemotherapy after the healing of surgical site in 2 weeks post operatively. Complete resolution of neurological deficits with significant improvement in gait was seen at the last follow-up 2 years after surgery. Repeat MRI revealed complete resolution of the disease.

Discussion RDD with spinal involvement is rare and it is often a challenge to arrive at the correct diagnosis. Histopathological characteristics and immunohistochemical findings are necessary for diagnosis. Resection of the lesion takes compression off the cord and therefore, is the primary line of management of this disease. Our case presentation serves as a reference when diagnosing and managing a patient of RDD.
\end{abstract}

\section{Introduction}

Rosai-Dorfman Disease (RDD) or sinus-histiocytosis primarily affects the lymph nodes $[1,2]$. Extra-nodal disease occurs in $30 \%$ of RDD, mostly involving bones, skin, and upper respiratory tract [3]. Central nervous system (CNS) is rarely involved in this disease $(<5 \%$ of extranodal RDD) and isolated spinal RDD is seen in $20-25 \%$ of CNS RDD [4, 5]. Spinal RDD predominantly affects children and young adults with a slight male preponderance. In this case report, we have described the successful management of isolated spinal RDD presenting with thoracic myelopathy.

S. Vidyadhara

vidya007@gmail.com

1 Spine Care Centre, Manipal Hospital, Bangalore, India

\section{Case report}

A 35-year-old male presented with upper back pain and progressive unsteadiness of gait of 2 months duration. $\mathrm{He}$ had tingling, numbness, and tightness of both lower limbs. He had gait imbalance and was unable to walk independently. He had stopped working which required him to go to his office. However, he was able to perform his activities of daily living, though with difficulty. He presented to us directly without having undergone any form of rehabilitative treatment. On examination, he was found to have incomplete spinal-cord injury with Grade D on ASIA Impairment Scale as he had hypoesthesia from T8 downwards and weakness over both the lower limbs (Grade-4/5 bilaterally as per MRC grading). Bowel and bladder functions were normal. Laboratory studies were unremarkable.

Contrast-MRI revealed an epidural lesion (hypo-intense on T1- and T2-weighted images) from C6-T7 causing severe spinal-cord compression (Fig. 1). He underwent laminectomy, excision of the lesion, and an instrumented posterolateral fusion from C6-T8. Intraoperatively, a firm, greyish-yellow, lobulated, well-encapsulated mass which 
Fig. 1 a Sagittal sections of T2-weighted MRI sequences revealed hypo-intense epidural lesion extending from C6-T7 levels causing severe spinal-cord compression. b Axial sections of T1-weighted MRI sequences at T3-T4 level showing an hypointense epidural lesion displacing the spinal cord to the left and causing severe compression. c Axial sections of STIR sequences showing a hyperintense large epidural lesion. d Coronal sections of MRI showing epidural lesion extending into neuro-foramen and causing compression over the spinal cord from the right side.
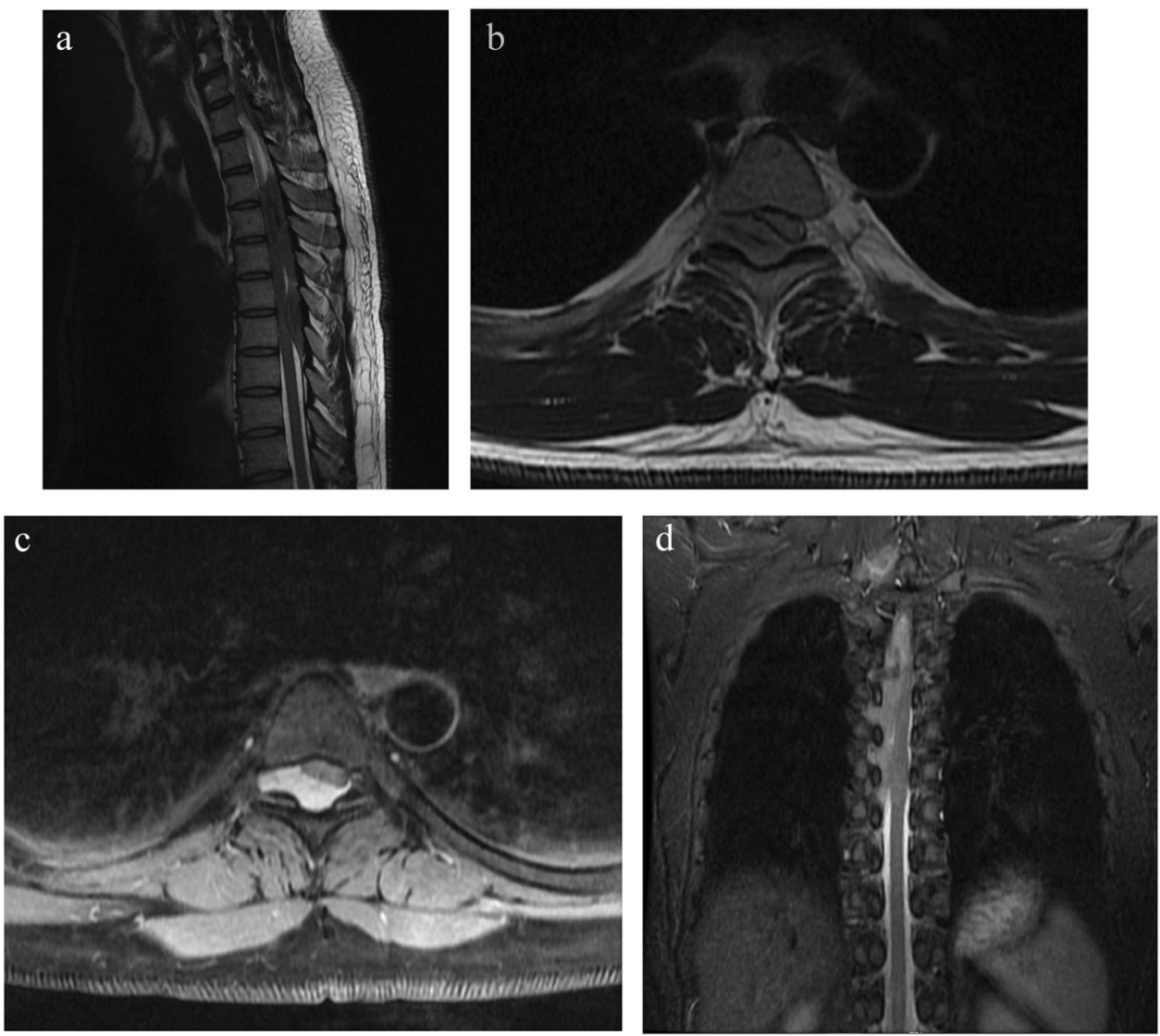

appeared to have a soft, "fish-flesh" appearance was excised. No intradural extension or bony involvement was detected. A clear demarcation between the tumor tissue and the surrounding anatomical structures could be appreciated intraoperatively which facilitated the complete resection of the tumor. However, as the tumor mass extended into the neuro-foramen, extensive bony resection including facetectomy was necessary. Hence, C6-T8 fusion was performed to prevent postoperative instability. Histopathological examination revealed inflammatory neoplastic tissue composed of clusters of large macrophages demonstrating emperipolesis (Fig. 2). There were clusters of plasma cells and lymphocytes dispersed over a fibrotic stroma. On immunohistochemistry, the macrophages were found to be CD68 and S-100 positive and CD1a negative. The plasma cells were observed to be polytypic on light-chain immunohistochemistry. Hence, the diagnosis of isolated spinal RDD was made.

There was no change in neurological examination post operatively. Mobilization was started on Post-op day 1 and muscle strengthening exercises for the lower limbs began. Two weeks post operatively, Inj. Vinblastin was started at a weekly dose of $10 \mathrm{mg}$ intravenously for a total duration of 7 months. At his 2-year follow-up, he ambulated independently and was independent in activities of daily living. $\mathrm{He}$ could walk for more than an hour and for more than $2 \mathrm{~km}$

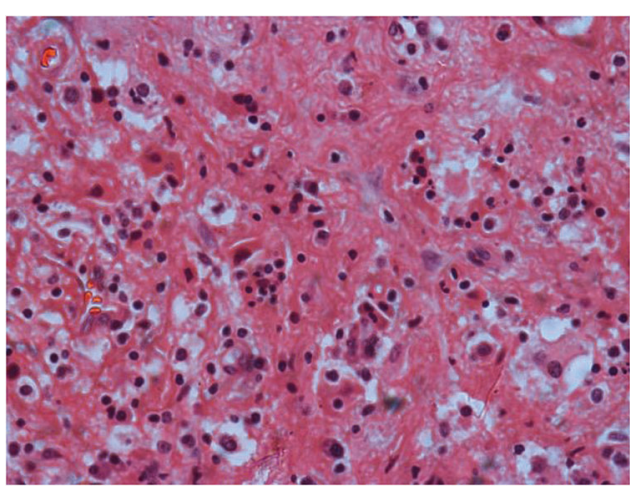

Fig. 2 Clusters of large macrophages demonstrating emperipolesis/ lymphagocytosis, which is a diagnostic criterion for RDD.

without the need for any physical assistance. He had resumed his job and had also started playing badminton as recreational activity. The repeat MRI confirmed complete resolution of the disease (Fig. 3).

\section{Discussion}

In this case report, we present a rare case of extra-nodal isolated spinal RDD with cord compression. In 1965, RDD was initially considered to be a lipid-storage disorder [6]. 
Fig. 3 a Sagittal section of repeat MRI performed 2 years later showing complete resolution of the RDD lesion. b Axial section of repeat MRI performed 2 years later showing complete resolution of the RDD lesion.
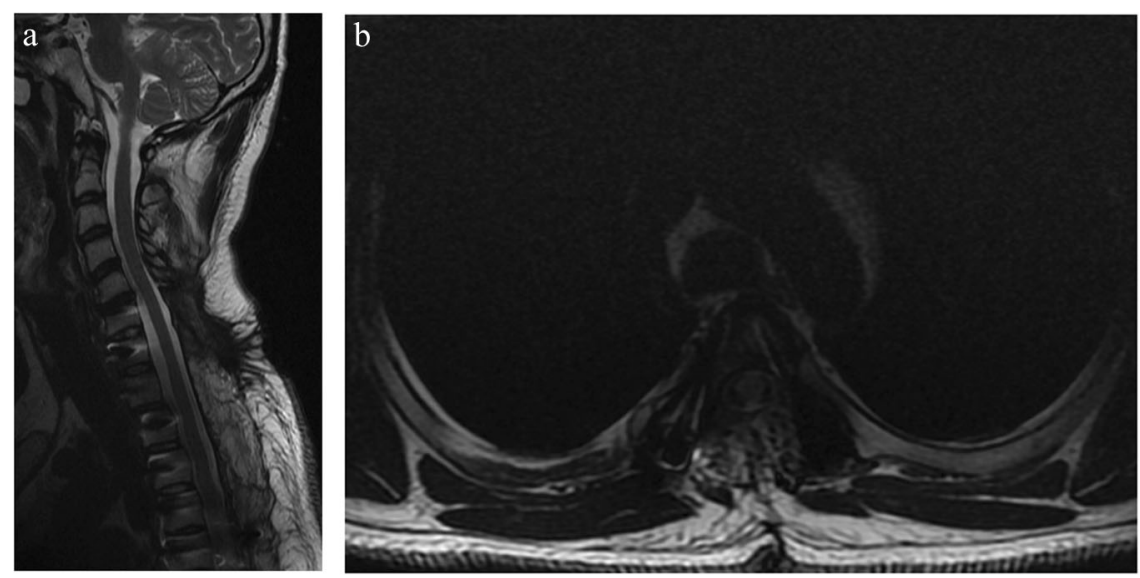

Later in 1969, Rosai and Dorfman described this disease as sinus histiocytosis with massive painless lymphadenopathy [1]. It is a self-limiting benign disease with unknown aetiology, which primarily involves the lymph nodes. However, different organ systems may be involved, with varied clinical presentation. Thus, histopathology remains the key to the diagnosis of this condition.

In the literature, isolated CNS RDD is seen in $<5 \%$ of all RDD cases [7, 8]. Moreover, spinal RDD in the absence of any other organ system involvement is even rarer [9]. Infections and unexplained immune system disorders have been considered as etiological factors for this disease $[10,11]$. According to some, $\operatorname{lgG} 4$ disorder has been considered as a possible cause [12-14]. However, in a previous study of nine RDD cases, they were unable to conclude that IgG4 played any role in the pathogenesis of this disease [14]. Our patient had normal IgG levels and hence this could not be considered the cause in our case.

Classical RDD presents with painless lymphadenopathy along with symptoms depending upon the location and size of the lesion [15]. Our patient presented with persistent upper back pain, paraparesis, and gait unsteadiness, secondary to spinal-cord compression at the cervicothoracic level.

The radiological findings of RDD are often nonspecific, making it a diagnostic dilemma. Extracranial-RDD lesions are usually isointense on T1-weighted imaging (T1WI) but mild hyperintensity is seen on T2-weighted imaging (T2WI) $[16,17]$. In our case, the lesion was hypo-intense on T1WI and T2WI. Nevertheless, it is often difficult to distinguish the MRI images of RDD from other more common extradural spinal lesions, like metastatic lesions, lymphomas, and plasma cell granulomas [17-20]. Also, literature does not show any characteristic MRI findings for RDD [21].

Identifying histopathological features of histiocytic proliferation with unusually large cells having large round/oval nuclei and nucleoli is the most definitive way of confirming
RDD. Emperipolesis/lymphagocytosis is a diagnostic criterion for RDD [2, 22]. The specific immunohistochemical features of RDD are the presence of CD68 and S-100 along with the absence of EMA and CD1a, which was confirmed in our case [23].

Treatment guidelines for RDD are variable. In general, lesions causing spinal-cord compression are preferably treated by its excision, which decompresses the cord and preserves the neurologic function [17, 18]. As spinal RDD is a benign disease, resection has shown excellent results with no recurrence $[16,24]$. A $14 \%$ recurrence rate was reported in a study of 29 patients followed for an average of 10.1 years [25]. Corticosteroids, radiotherapy, and chemotherapy have been recommended for recurrence [21, 26, 27]. In our case, Vinblastin treatment for 7 months following excision led to complete resolution of symptoms and significant improvement in gait at 2 years.

RDD has a good prognosis, with mortality primarily related to intracranial RDD [28]. Timely administration of multi-modality treatment can prevent morbidity from this disease.

\section{Conclusion}

Isolated spinal RDD is extremely rare and difficult to diagnose. Resection of the lesion is the primary treatment. The etiology and optimal adjuvant treatments require further research.

\section{Compliance with ethical standards}

Conflict of interest The authors declare that they have no conflict of interest.

Publisher's note Springer Nature remains neutral with regard to jurisdictional claims in published maps and institutional affiliations. 


\section{References}

1. Rosai J, Dorfman RF. Sinus histocytosis with massive lymphadenopathy. A newly recognized benign clinicopathological entity. Arch Pathol. 1969;87:63-70.

2. Al-Saad K, Thorner P, Ngan B, Gerstle J, Kulkarni A, Babyn P, et al. Extranodal Rosai-Dorfman disease with multifocal bone and epidural involvement causing recurrent spinal cord compression. Pediatr Dev Pathol. 2005;8:593-8.

3. Symss NP, Cugati G, Vasudevan MC, Ramamurthy R, Pande A. Intracranial Rosai-Dorfman disease: report of three cases and literature review. Asian J Neurosurg. 2010;5:19-30.

4. Foucar E, Rosai J, Dorfman R. Sinus histiocytosis with massive lymphadenopathy (Rosai-Dorfman disease): review of the entity. Semin Diagn Pathol. 1990;7:19-73.

5. Hargett C, Bassett T. Atypical presentation of sinus histiocytosis with massive lymphadenopathy as an epidural spinal cord tumor: a case presentation and literature review. J Spinal Disord Tech. 2005; 18:193-6.

6. Destombes P. Adenitis with lipid excess, in children or young adults, seen in the Antilles and in Mali. (4 cases). Bull Soc Pathol Exot Filiales. 1965;58:1169-75.

7. Wu M, Anderson AE, Kahn LB. A report of intracranial Rosai-Dorfman disease with literature review. Ann Diagn Pathol. 2001;5:96-102.

8. Siadati A, Powell SZ, Shahab I, Valadka AB, Parker JR. Pathologic quiz case: a 48 year-old woman with a dural-based intracranial tumor. Arch Pathol Lab Med. 2001;125:1115-6.

9. Raslan O, Ketonen LM, Fuller GN, Schellingerhout D. Intracranial Rosai-Dorfman disease with relapsing spinal lesions. J Clin Oncol. 2008;26:3087-9.

10. Castionia J, Mihaescu A, So AK. Sinus histiocytosis with massive lymphadenopathy (Rosai-Dorfman Disease) and oligoarthritis. Jt Bone Spine. 2011;78:641-3.

11. Nathan B, Roomallah B, Salisbury JR, Tenant-Flowers M, Kassam S. A rare cause of lymphadenopathy-Rosai-Dorfman disease in a HIV-positive Ugandan woman. Int J STD AIDS. 2016;27:1123-5.

12. Roberts SS, Attanoos RL. IgG4+ Rosai-Dorfman disease of the lung. Histopathology. 2010;56:662-4.

13. de Jong W, Kluin P, Groen H. Overlapping immunoglobulin G4related disease and Rosai-Dorfman disease mimicking lung cancer. Eur Respir Rev. 2012;21:365-7.
14. Wang Q, Gan M, Weng S, Zhou T, Zheng H, Chen H, et al. Expression of IgG4 in Rosai-Dorfman disease and its significance. Chin J Pathol. 2015;44:729-33.

15. Bhandari A, Patel PR, Patel MP. Extranodal Rosai-Dorfman disease with multiple spinal lesions: a rare presentation. Surg Neurol. 2006;65:308-11.

16. Adeleye AO, Amir G, Fraifeld S, Shoshan Y, Umansky F, Spektor S. Diagnosis and management of Rosai-Dorfman disease involving the central nervous system. Neurol Res. 2010;32:572-8.

17. Xu H, Zhang F, Lu F, Jiang J. Spinal Rosai-Dorfman disease: case report and literature review. Eur Spine J. 2017;26:117-27.

18. Osenbach R. Isolated extranodal sinus histiocytosis presenting as an intramedullary spinal cord tumor with paraplegia. J Neurosurg. 1996;85:692-6.

19. Abla O, Jacobsen E, Picarsic J, Krenova Z, Jaffe R, Emile JF, et al. Consensus recommendations for the diagnosis and clinical management of Rosai-Dorfman-Destombes disease. Blood. 2018;131:2877-90.

20. $\mathrm{Wu} \mathrm{L}, \mathrm{Xu} \mathrm{Y}$. Rosai-Dorfman disease: a rare lesion with dura tail sign mimicking spinal meningioma. Spine J. 2014;14:3058-9.

21. $\mathrm{Li} \mathrm{Y}$, Wang $\mathrm{X}, \mathrm{Gao} \mathrm{J}, \mathrm{Yu} \mathrm{S}, \mathrm{Li} \mathrm{Z}$. Isolated extradural Rosai-Dorfman disease causing the spinal cord compression. Medicine. 2018;97:e12722.

22. Govender D, Chetty R. Inflammatory pseudotumor and Rosai-Dorfman disease of soft tissue: a histological continuum? J Clin Pathol. 1997;50:79-81.

23. Tu J, Li W, Yang C. Rosai-Dorfman disease of the subdural spine with a long segment lesion: a case report and literature review. J Int Med Res. 2017;45:875-81.

24. Lopez P, Estes ML. Immunohistochemical characterization of the histiocytes in sinus histiocytosis with massive lymphadenopathy: analysis of an extranodal case. Hum Pathol. 1989;20:711-5.

25. Petzold A, Thom M, Powell M, Plant GT. Relapsing intracranial Rosai-Dorfman disease. J Neurol Neurosurg Psychiatry. 2001;71:538-41.

26. Komp DM. The treatment of sinus histiocytosis with massive lymphadenopathy (Rosai-Dorfman disease). Semin Diagn Pathol. 1990;7:83-6.

27. Kidd DP, Revesz T, Miller NR. Rosai-Dorfman disease presenting with widespread intracranial and spinal cord involvement. Neurology. 2006;67:1551-5.

28. Andriko JA, Morrison A, Colegial CH, Davis BJ, Jones RV. Rosai-Dorfman disease isolated to the central nervous system: a report of 11 cases. Mod Pathol. 2001;14:172-8. 